\title{
Rôles potentiels de la symbiose mycorhizienne dans la conservation des populations méditerranéennes de Genévrier thurifère (Juniperus thurifera L.)
}

\author{
Mycorrhizal symbiosis potentialities to improve the management \\ performances of Juniperus thurifera L. populations \\ in Mediterranean areas
}

H. SANGUIN ${ }^{1}$, S. KHOULISSA ${ }^{2}$, L. ZARIK ${ }^{2}$, H. GRYTA ${ }^{3,4}$, A. BOUMEZZOUGH ${ }^{2}$, L. OUAHMANE ${ }^{2}$, M. HAFIDI ${ }^{2}$, A. OUHAMMOU ${ }^{2}$, Y. PRIN ${ }^{1}$, B. DREYFUS ${ }^{5}$, J. CAMBECÈDES ${ }^{6}$, L. MICHÉ7 ${ }^{7}$ T. GAUQUELIN ${ }^{8}$ \& R. DUPONNOIS ${ }^{5, *}$

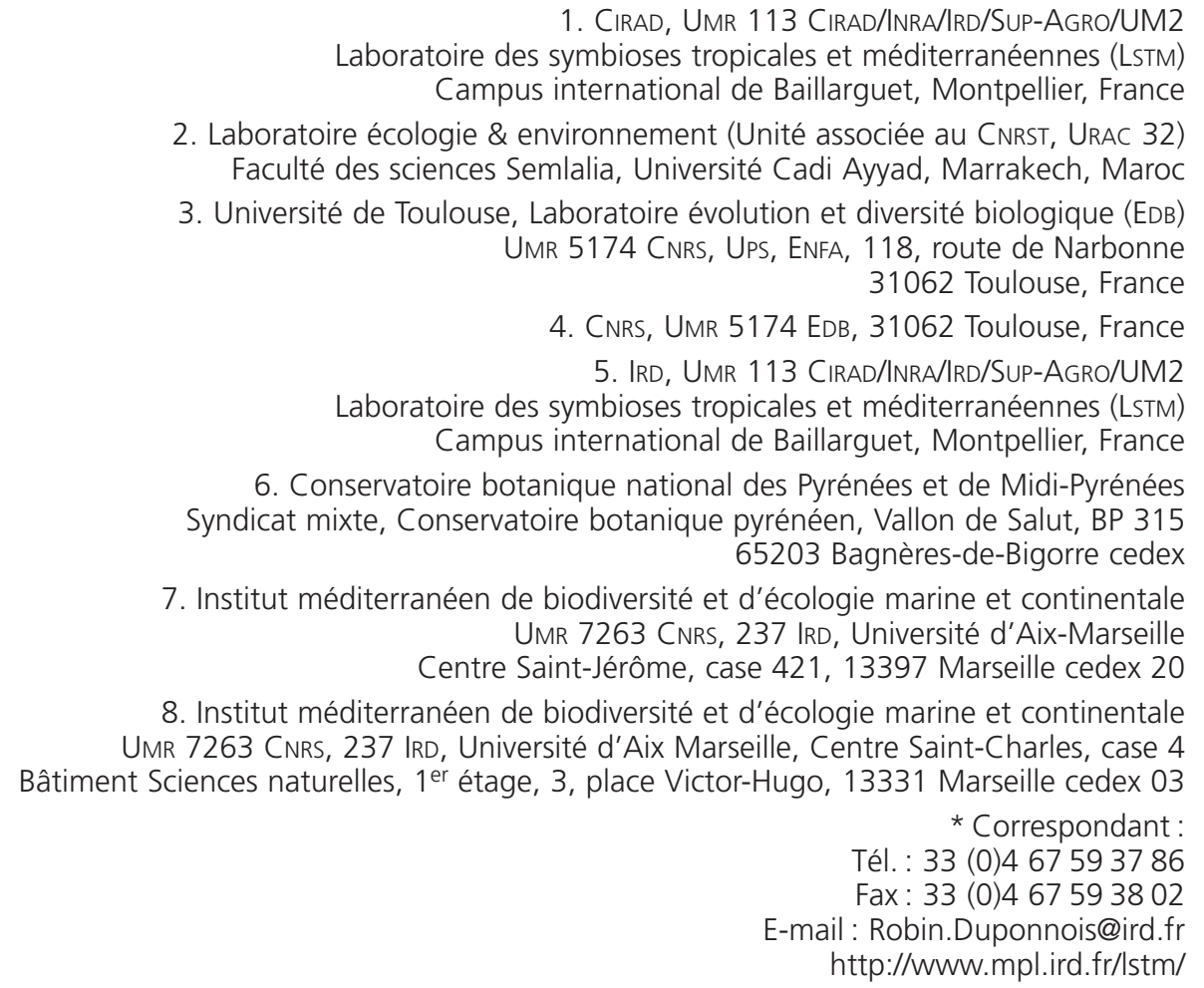

\section{Résumé}

Cet article de synthèse a pour principaux objectifs de rassembler des connaissances concernant le statut mycorhizien des espèces du genre Juniperus et plus particulièrement celui de J. thuri-

Mots clés : Cupressaceae, Juniperus, symbiose mycorhizienne, plantes nurses, diversité, revégétalisation. fera. Les résultats présentés mettent en exergue le manque évident de connaissances scientifiques sur le rôle de la symbiose mycorhizienne dans l'écologie de cette essence forestière. Toutefois et malgré ces lacunes, quelques observations permettent d'envisager une valorisation

Keywords: Cupressaceae, Juniperus, mycorrhizal symbiosis, nurse plant, diversity, afforestation programme. 
performante de la symbiose mycorhizienne dans les opérations de gestion des formations à Juniperus que ce soit via la mycorhization contrôlée ou la gestion de la flore accompagnatrice en identifiant les plantes nurses les plus efficaces. Des exemples de validation de ces techniques d'ingénierie écologique sont présentés avec une autre Cupressacée, le Cyprès de l'Atlas, en soulignant les possibilités de transfert au modèle Juniperus. Afin de valoriser au mieux la symbiose mycorhizienne dans la mise en place de plan de gestion des formations à Juniperus, il sera nécessaire d'acquérir des connaissances beaucoup plus conséquentes au niveau de la structure du cortège mycorhizien associé à J. thurifera et de son rôle écologique dans les différents stades de développement de l'arbre.

\begin{abstract}
This synthesis has as main objectives to gather knowledge concerning the mycorrhizal status of Juniperus species and more particularly that of J. thurifera. The results highlight the obvious lack of scientific knowledge on the role of mycorrhizal symbiosis in the ecology of this forest species. However, despite these scientific gaps, some observations can be considered to introduce the mycorrhizal symbiosis in afforestation programs with Juniperus either through controlled mycorrhization or accompanying vegetation management by identifying the most effective nurse plants. Examples of validation of these ecological engineering techniques are presented with another Cupressacée, Cupressus atlantica, highlighting the possibilities of transferring these cultural techniques to the model Juniperus. To ensure a positive impact of the mycorrhizal symbiosis in Juniperus management plans, it is necessary to learn much more concerning the structure of the mycorrhizal communities associated with J. thurifera and their ecological roles in the different stages of tree development.
\end{abstract}

\section{Introduction}

La désertification désigne la dégradation des terres dans les zones arides, semi-arides et subhumides consécutive aux impacts conjugués des activités humaines et, dans une moindre mesure, des crises climatiques (Francis \& Thornes 1990). Les premiers symptômes de cette dégradation sont enregistrés au niveau du couvert végétal avec une altération de sa densité et de sa composition floristique avec pour principales conséquences, une baisse significative des capacités de reproduction et de régénération des espèces végétales les plus exploitées (exemple : essences forestières). De plus et du fait que les sols ne bénéficient plus du rôle protecteur de la strate végétale, ces derniers sont fragilisés et soumis aux effets de l'érosion hydrique et éolienne entraînant à terme une diminution des teneurs en éléments minéraux, en matière organique, de la stabilité structurale et de la porosité du sol (Garcia et al. 1997 ; Albaladejo et al. 1998). Ce processus de désertification engendre également des modifications au niveau des caractéristiques génétiques et fonctionnelles de la microflore du sol aboutissant à des dysfonctionnements dans le déroulement des principaux cycles biogéochimiques telluriques $(\mathrm{N}, \mathrm{P})$ et ainsi une baisse globale de la fertilité du sol (Requena et al. 2001). Parmi les composantes microbiennes particulièrement affectées par ces dégradations figurent les champignons mycorhiziens. L'organe appelé «mycorhizes » résulte d'une union durable entre les racines de la majorité des végétaux et certains champignons symbiotiques du sol, basée sur des échanges réciproques, et constitue un élément essentiel dans le continuum sol/plantes/microorganismes. En effet, la majeure partie des espèces végétales ne peut se développer sans l'établissement d'une symbiose mycorhizienne fonctionnelle au sein de leur système racinaire (Smith \& Read 2008). La composante fongique de cette association symbiotique améliore significativement le développement de la plante dans des conditions de carences minérales (plus particulièrement en phosphore, principale carence enregistrée dans les sols dégradés méditerranéens, au même titre que l'azote), de déficit hydrique et permet également à la plante de mieux résister aux attaques de pathogènes (Barea et al. 1997 ; Schreiner et al. 1997 ; Bethlenfalvay \& Linderman 1992). La symbiose mycorhizienne joue aussi un rôle majeur dans les mécanismes biologiques régissant l'évolution spatio-temporelle, la diversité spécifique, la stabilité et la productivité des écosystèmes végétaux terrestres (Odum 1959; Van der Heijden et al. 1998). La maîtrise de la symbiose mycorhizienne et son introduction dans les itinéraires sylvicoles apparaît donc comme un outil performant susceptible d'améliorer les performances des opérations de reboisement de sites dégradés mais également de conserver et valoriser une biodiversité en ressources ligneuses en optimisant la productivité et la stabilité de formations forestières fragilisées par une surexploitation excessive consécutive à une pression anthropique exacerbée. 
Le Genévrier thurifère (Juniperus thurifera L.) appartient à la famille des Cupressacées et est considéré comme une espèce emblématique des montagnes du bassin ouest-méditerranéen. Dans le nord du pourtour méditerranéen (Italie, France, Espagne) et jusqu'au début des années 1960, les formations de Genévrier ont été particulièrement réduites, voire décimées, et ce n'est que relativement récemment que les programmes de conservation ont permis de contribuer à la protection durable des thuriféraies. Par contre, en Afrique du Nord (Algérie, Maroc), cette essence forestière est considérée comme menacée d'extinction du fait principalement d'un impact anthropique sévère (pratiques culturales inadaptées, pression pastorale, etc.) qui limite les processus de régénération naturelle de l'espèce et en conséquence accélère le vieillissement des peuplements (Gauquelin et al. 1999). Au Maroc, le Genévrier thurifère est considéré comme l'essence forestière ayant la plus régressée en affichant un recul de $90 \%$ par rapport à son aire potentielle de répartition. Dans ces régions, cette espèce forme des peuplements clairs sur des terrains dénudés et rocheux (figure 1). Dans ces conditions drastiques où les phénomènes d'érosion diminuent la fertilité des sols conjugués à une surexploitation de cette ressource ligneuse et une forte pression pastorale, cette situation apparaît comme un terrain privilégié pour valoriser les propriétés de la symbiose mycorhizienne au profit du développement durable de ces thuriféraies en parallèle à la mise en œuvre de mesures visant à sauvegarder ces écosystèmes et à sensibiliser les populations à la nécessité de cette conservation. Toutefois les connaissances portant sur le statut mycorhizien de $J$. thurifera et son impact sur le développement de cette essence (plus particulièrement au niveau des stades juvéniles) restent encore très insuffisantes pour pouvoir valoriser cette symbiose au sein de plans de conservation de cet arbre, plus particulièrement en Afrique du Nord.

Les principaux objectifs de cet article de synthèse sont : (1) de présenter les connaissances scientifiques décrivant le statut mycorhizien de $J$. thurifera et (2) de proposer des stratégies d'ingénierie écologique, déjà validées pour d'autres essences forestières méditerra-

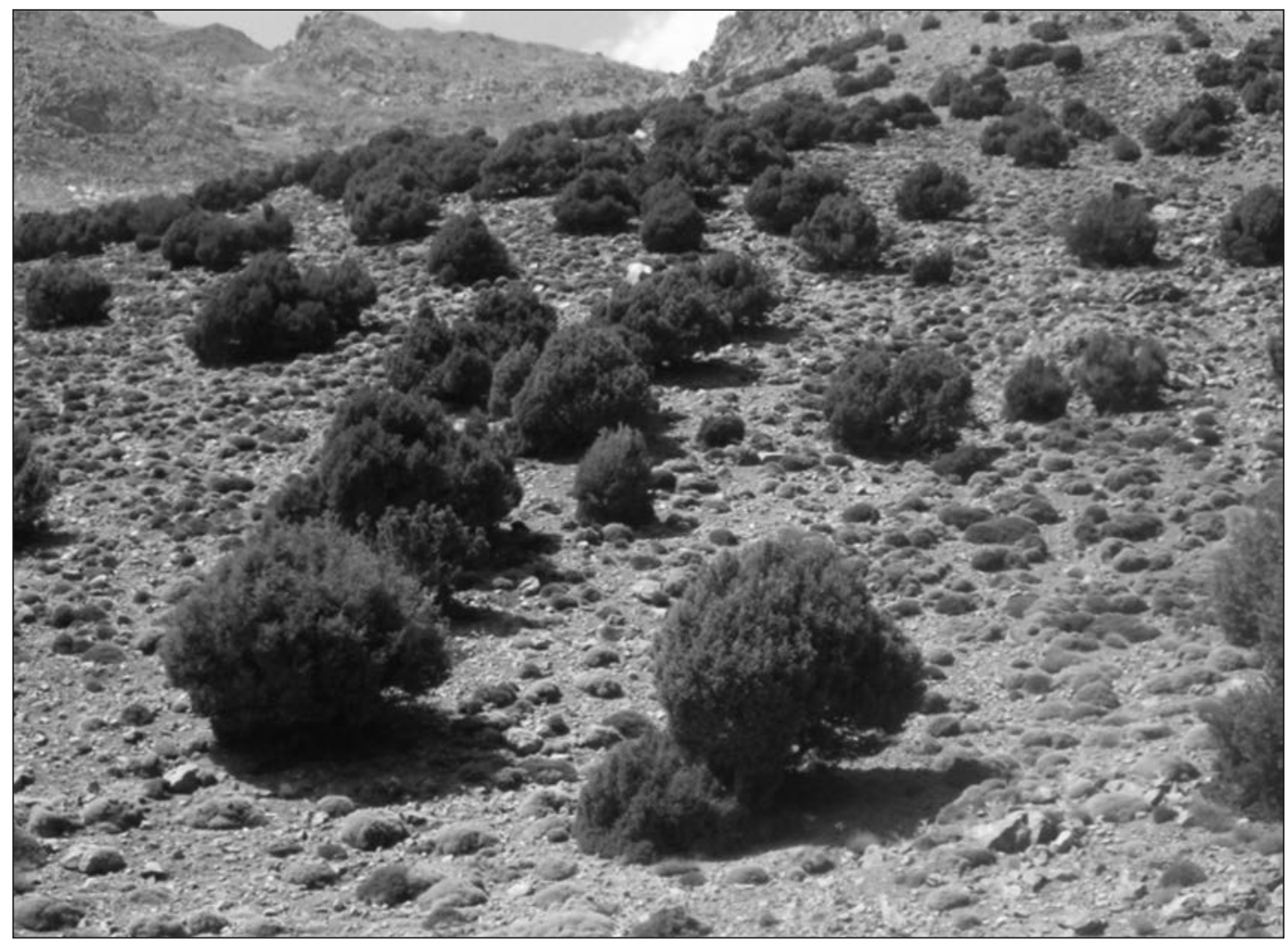

Photo: Ali Boumezzough

Figure 1 - Peuplement de Juniperus thurifera de Tizrag (Haut Atlas central) localisée à 2700 m d'altitude et à $75 \mathrm{~km}$ au sud de Marrakech (Maroc).

Figure 1 - Stand of Juniperus thurifera at Tizrag (Central High Atlas) located at 2,700 m altitude and $75 \mathrm{~km}$ south of Marrakech (Morocco). 
néennes telles que le Cyprès de l'Atlas (Duponnois et al. 2011), afin de valoriser la symbiose mycorhizienne comme outil biologique favorisant le développement de cette espèce forestière dans des conditions hostiles (carences en éléments nutritifs, déficit hydrique, etc.) en favorisant plus particulièrement les processus écologiques impliqués dans la régénération naturelle de cette espèce.

\section{Le statut mycorhizien de J. thurifera}

Les données disponibles sur le statut mycorhizien des espèces appartenant au genre Juniperus sont relativement éparses dans la littérature scientifique. Le tableau 1 récapitule les principaux types de symbioses mycorhiziennes observés chez ce genre. Les deux types principaux de symbiose mycorhizienne (symbiose ectomycorhizienne et symbiose mycorhizienne à arbuscules) ont été fréquemment observés au niveau des racines des différentes espèces de Juniperus étudiées. Cette double symbiose est détectée chez certaines essences forestières comme Alnus (Molina et al. 1994), Populus (Lodge 1989; Lodge \& Wentworth 1990), Salix (Lodge 1989; Dhillion 1994), Eucalyptus (Lapeyrie \& Chilvers 1985 ; Adjoud-Sadadou \& HalliHargas 2000) et Acacia (Founoune et al.

Tableau 1 - Principaux types de symbioses mycorhiziennes observés chez les espèces du genre Juniperus.

Table 1 - Main types of mycorrhizal symbioses reported in the Juniperus genus.

\begin{tabular}{|lcc|}
\hline Espèces & \multicolumn{2}{c|}{ Statut mycorhizien } \\
\hline & Endotrophe & Ectotrophe \\
J. communis L. & $\mathrm{x}$ & $\mathrm{x}$ \\
J. nana Willd (J. communis spp. nana) & & $\mathrm{x}$ \\
J. scopularum Sarg. & $\mathrm{x}$ & $\mathrm{x}$ \\
J. virginia L. & & $\mathrm{x}$ \\
J. macrocarpa Sibth. \& Sm & & $\mathrm{x}$ \\
J. vulgaris Ray. & & $\mathrm{x}$ \\
J. rigida Sied. \& Zucc. & & $\mathrm{x}$ \\
J. thurifera L. & $\mathrm{x}$ & $\mathrm{x}$ \\
J. chinensis L. & $\mathrm{x}$ & \\
J. monosperma Sarg. & $\mathrm{x}$ & \\
J. occidentalis Hook. & $\mathrm{x}$ & \\
J. osteosperma Little & $\mathrm{x}$ & \\
J. oxycedrus L. & $\mathrm{x}$ & \\
J. phoenica L. & $\mathrm{x}$ & \\
J. procera Hochst & $\mathrm{x}$ & \\
J. sabina L. & $\mathrm{x}$ & \\
& & \\
\hline
\end{tabular}

2002). Elle a également été signalée chez des espèces supposées «ectotrophes strictes » comme Abies (Cázares \& Trappe 1993), Pseudotsuga et Tsuga (Cázares \& Smith 1996). La présence de ces types de symbioses mycorhiziennes permet principalement à la plante hôte de mieux utiliser les ressources du milieu en fonction du stade d'évolution du couvert arboré mais également au cours de la saison en fonction des conditions climatiques (Van der Heijden \& Vosatka 1999; Van der Heijden 2001). Les facteurs biotiques et abiotiques régissant cette succession dans la composition du cortège mycorhizien associé à certaines espèces de Juniperus et son impact sur le développement de la plante selon les caractéristiques environnementales ont été rarement étudiés. Concernant J. thurifera, des travaux ont été entrepris par Gryta \& Gardes (résultats non publiés) afin de (1) décrire le statut mycorhizien de cette espèce, (2) déterminer la dynamique de la mycorhization au cours du développement du Genévrier et sa dépendance vis-à-vis d'un type de symbiose en fonction de l'âge de l'arbre et (3) rechercher une dynamique annuelle saisonnière de la mycorhization. Les résultats ont confirmé la présence des deux types de symbiose chez J. thurifera. Les taux de mycorhization par les champignons à arbuscules et les champignons ectomycorhiziens étaient particulièrement élevés suggérant ainsi l'importance de la symbiose mycorhizienne dans le développement de l'arbre. La présence de structures mycorhiziennes a été détectée quel que soit l'âge des individus et il a pu être démontré que l'intensité de l'ectomycorhization des racines augmentait avec l'âge de l'arbre alors que cette tendance n'a pas été observée avec les champignons mycorhiziens à arbuscules. Une dynamique saisonnière de l'établissement de la symbiose ectomycorhizienne a également été décrite. Les ectomycorhizes se forment au printemps (fin avril-début mai) sur de jeunes racines, se nécrosent ensuite pendant la sécheresse estivale puis dégénèrent en hiver. Concernant les champignons à arbuscules, cette dynamique de colonisation n'a pas été observée mais, par contre, leur activité est variable en fonction de la saison avec des pics au printemps et en automne. Ces variations saisonnières dans les caractéristiques de la colonisation et de l'activité mycorhizienne sont à mettre en relation avec les rythmes d'activité des racines montrant ainsi l'importance de la symbiose mycorhizienne pour le développement de la plante. 


\section{Exemples de stratégies de gestion du potentiel mycorhizien des sols pour optimiser le développement de Juniperus spp.}

Dans la littérature, il n'existe que très peu de données permettant d'établir la dépendance mycorhizienne des espèces du genre Juniperus. La dépendance mycorhizienne représente l'impact de l'établissement de la symbiose mycorhizienne sur la croissance de la plante par rapport à celle de la plante non mycorhizée (Plenchette et al. 1983). Roldan et al. (2006) ont montré que l'inoculation d'un complexe de champignons mycorhiziens à arbuscules (Glomus intraradices, G. deserticola et G. mosseae) stimulait significativement la croissance de jeunes plants de J. oxycedrus en conditions contrôlées. Cet effet positif de l'inoculation était accompagné par une amélioration significative de la nutrition en phosphore de la plante. Ils ont ainsi calculé que, après quatorze mois de culture, la dépendance mycorhizienne de J. oxycedrus était de 57,8\%, valeur comparable à celles obtenues avec des légumineuses dont la croissance est étroitement liée à l'établissement de la symbiose mycorhizienne (Duponnois et al. 2001). En conséquence, cette espèce de Juniperus peut être considérée comme hautement dépendante de la symbiose mycorhizienne pour lui assurer une croissance optimale (Habte \& Manjunath 1991). Toutefois, il n'existe pas à notre connaissance de résultats mettant en exergue l'importance de l'inoculation mycorhizienne dans le cadre d'opérations de reboisement ou de gestion durable des formations à Juniperus.
En foresterie, deux principales stratégies d'ingénierie écologique peuvent être entreprises afin d'améliorer le potentiel mycorhizien des sols et ainsi permettre à la symbiose mycorhizienne de développer ses propriétés au profit du développement de la plante hôte: (1) la «mycorhization contrôlée» qui repose sur l'identification de souches fongiques performantes quant à leur effet sur un paramètre donné (exemple : effet sur la croissance de la plante hôte) qui seront ensuite inoculées en masse dans le substrat de culture au cours de la phase de culture en pépinière pour ensuite transplanter les plants mycorhizés dans le site à reboiser (Duponnois et al. 2007) ; (2) l'adoption de pratiques culturales (exemple : utilisation de plantes nurses) pour améliorer le potentiel mycorhizien des sols en favorisant l'abondance et la diversité des propagules mycorhiziennes du site à revégétaliser (Ouahmane et al. 2006). Ces pratiques ont été testées expérimentalement au Maroc avec une autre Cupressacée, Cupressus atlantica (Ouahmane et al. 2007 ; Duponnois et al. 2011).

\section{La mycorhization contrôlée du Cyprès de l'Atlas (Cupressus atlantica)}

Une expérience de mycorhization contrôlée a été mise en place dans la vallée de NFis ( $\left.8^{\circ} 17^{\prime} 02^{\prime \prime} \mathrm{W}, 31^{\circ} 54^{\prime} 34^{\prime \prime} \mathrm{N}\right)$ en utilisant des jeunes plants de Cyprès préalablement inoculés par un complexe de champignons mycorhiziens à arbuscules. Les résultats ont montré que, après six mois de culture en pépinière, les plants mycorhizés présentaient une croissance significativement plus importante que celle des plants non mycorhizés (tableau 2).

Tableau 2 - Effet de l'inoculation par un complexe de champignons mycorhiziens sur la croissance et la nutrition minérale de plants de Cyprès de l'Atlas (Cupressus atlantica) après 6 mois de culture en pépinière. D'après Ouahmane et al. (2007).

Table 2 - Effect of the mycorrhizal inoculation on the growth and mineral nutrition of Cypress plants (Cupressus atlantica) after 6 months of culture in nursery conditions. From Ouahmane et al. (2007).

\begin{tabular}{|c|c|c|}
\hline \multirow[t]{2}{*}{ Paramètres mesurés } & \multicolumn{2}{|c|}{ Traitements } \\
\hline & Témoin & + Complexe mycorhizien \\
\hline Hauteur $(\mathrm{cm})$ & $12,3(0,46)^{1} a^{2}$ & $16,1(0,71) b$ \\
\hline Biomasse aérienne (mg poids sec) & $1163(20) \mathrm{a}$ & $1893(264) b$ \\
\hline Biomasse racinaire (mg poids $\mathrm{sec}$ ) & $740(64) a$ & $1183(132) b$ \\
\hline Biomasse totale (mg poids sec) & $1903(62) a$ & $3077(392) b$ \\
\hline Teneur en $\mathrm{P}$ des parties aériennes ( $\mathrm{mg} \mathrm{g}^{-1}$ poids sec) & $0,09(0,01) a$ & $0,167(0,03) b$ \\
\hline Teneur en $\mathrm{N}$ des parties aériennes ( $\mathrm{mg} \mathrm{g}^{-1}$ poids sec) & $2,23(0,23) a$ & $3,50(0,1) b$ \\
\hline Taux de mycorhization (\%) & - & $41,2(2,3)$ \\
\hline
\end{tabular}




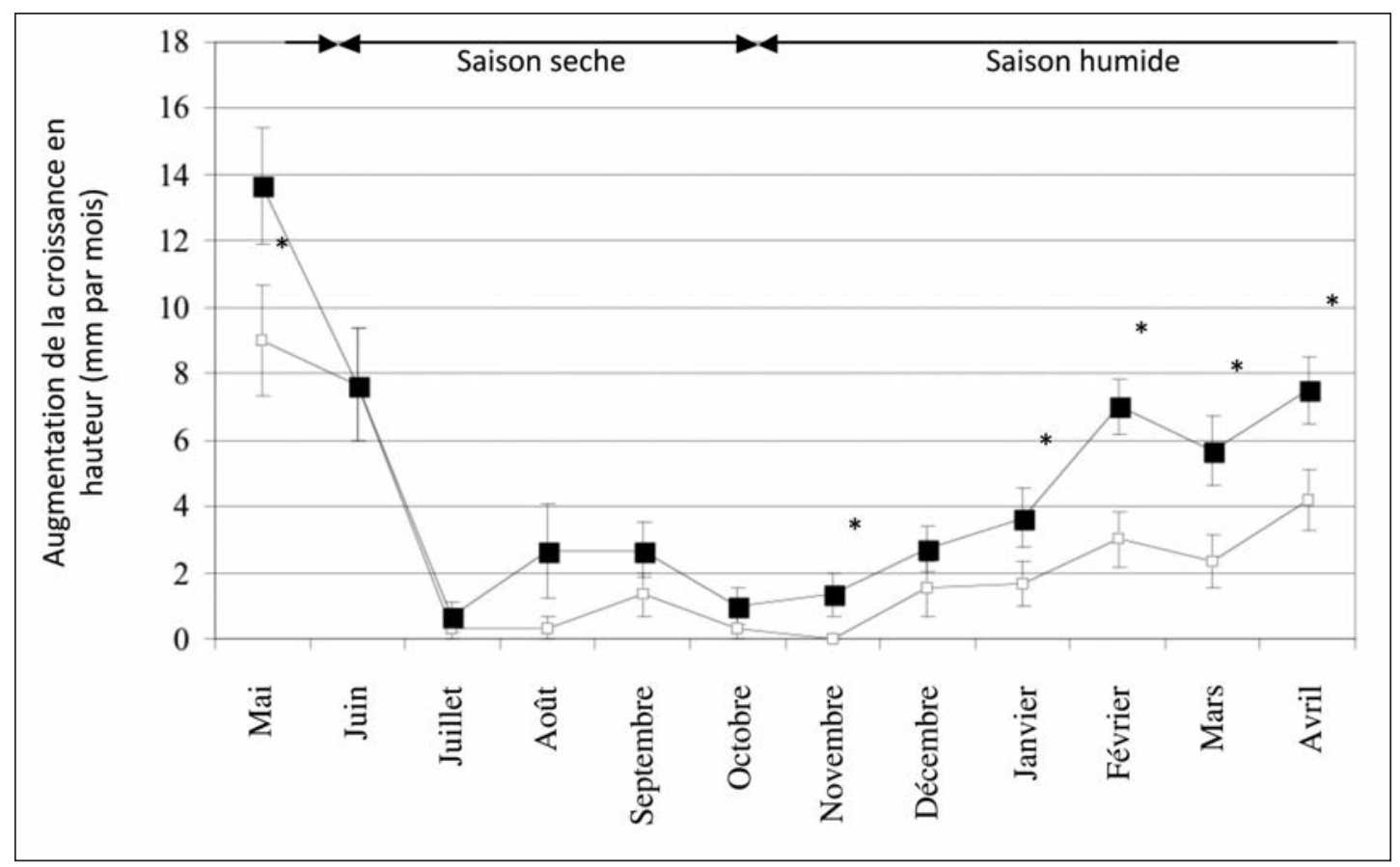

Figure 2 - Évolution de la croissance en hauteur des plants de Cyprès de l'Atlas au cours de la première année de plantation (d'après Ouahmane et al. 2007). $\square$ Plants mycorhizés, $\square$ Plants non mycorhizés. * : Différence significative d'après le test de Newman-Keul $(p<0,05)$.

Figure 2 - Evolution of the height growth of seedlings Cypress Atlas during the first year of plantation (From Ouahmane et al. 2007). Mycorrhized plants, $\square$ Non mycorrhized plants. * : Significantly different according to the Newman-Keul's test $(p<0.05)$.
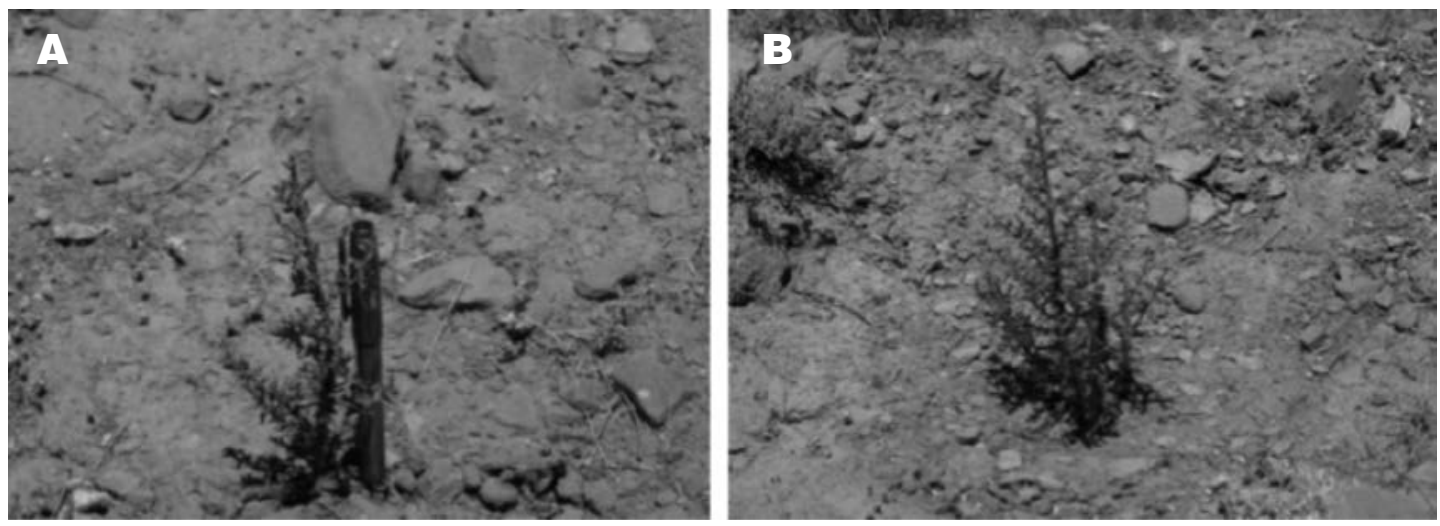

Photos: Robin Duponnois

Figure 3 - Plants de Cyprès de l'Atlas inoculés (B) ou non inoculés (A) après une année de plantation en milieu naturel. D'après Ouahmane et al. (2007).

Figure 3 - Cypress Plants not inoculated $(A)$ or inoculated with mycorrhizal fungi $(B)$ after one year of plantation in field conditions. From Ouahmane et al. (2007).

Lors du transfert au champ, la mortalité des plants mycorhizés était beaucoup plus faible $(17 \%)$ que celle enregistrée chez les plants non mycorhizés $(37 \%)$. L'inoculation mycorhizienne a stimulé la croissance des plants ainsi que leur nutrition minérale (figures 2, 3, tableau 3). Il est à noter que les plants mycorhizés ont connu une meilleure reprise de croissance en fin de saison sèche suggérant la capacité des plants inoculés à utiliser de manière optimale la ressource en eau des sols.

\section{La gestion du potentiel mycorhizien des sols par la valorisation des ressources végétales pionnières: exemple des plantes nurses}

Dans les écosystèmes méditerranéens, la strate arbustive est organisée sous forme de «poches » de végétation qui matérialisent des îlots de fertilité chimique et biologique au niveau du sol (Garner \& Steinberger 1989). Ce type de végétation améliore l'infiltration 
Tableau 3 - Effet de l'inoculation mycorhizienne sur la nutrition minérale des plants de Cyprès de l'Atlas après un an de plantation. D'après Ouahmane et al. (2007).

Table 3 - Effect of the mycorrhizal inoculation on the mineral nutrition of Cypress plants after one year of plantation. From Ouahmane et al. (2007).

\begin{tabular}{lrr|}
\hline Paramètres mesurés & \multicolumn{2}{c|}{ Traitements } \\
\hline & Témoin & + Complexe mycorhizien \\
\hline Teneur en N des parties aériennes $\left(\mathrm{mg} \mathrm{g}^{-1}\right.$ poids sec) & $2,01(0,13)^{1} \mathrm{a}^{2}$ & $3,66(0,19) \mathrm{b}$ \\
Teneur en P des parties aériennes $\left(\mathrm{mg} \mathrm{g}^{-1}\right.$ poids sec) & $0,077(0,004) \mathrm{a}$ & $0,114(0,004) \mathrm{b}$ \\
\hline $\begin{array}{l}\text { 1. Erreur standard. } \\
\text { 2. Les valeurs d'une même ligne suivies par une même lettre ne sont pas significativement différentes d'après le test de } \\
\text { Newman-Keul ( } \mathrm{p}<0,05) \text {. }\end{array}$ \\
\hline
\end{tabular}

de l'eau dans le sol, la teneur en matière organique, etc. (Valladares \& Pugnaire 1999) mais a également un impact positif sur la régénération naturelle des ressources forestières du milieu (Bashan et al. 2009). Cette interaction positive interspécifique est généralement expliquée par les caractéristiques abiotiques du microenvironnement qu'offrent ces îlots de fertilité aux jeunes régénérations (faibles variations de température, régulation de l'humidité du sol, etc.). Plus récemment, il a été démontré que les espèces arbustives impliquées facilitaient la multiplication des propagules mycorhiziennes et ainsi contribuaient au maintien d'un haut potentiel mycorhizien susceptible d'améliorer le développement du jeune plant de l'essence considérée (Duponnois et al. 2011). Après avoir identifié les espèces arbustives accompagnatrices du Cyprès de l'Atlas dans la vallée de NFis (Haut Atlas, Maroc), des essais de coculture C. atlantica/Lavandula stoechas ont été mis en place. Les résultats ont montré que la présence de la Lavande à proximité de jeunes plants de Cyprès stimulait la croissance de l'arbre mais également favorisait le biofonctionnement du sol (Duponnois et al. 2011). Ces arbustes (appelés également «plante nurse » en raison de leur impact sur la jeune régénération de l'essence forestière) pourraient donc constituer des vecteurs de propagation de symbiotes fongiques dans le sol et assurer ainsi un maintien durable de la productivité et de la stabilité de la plantation. Il a été suggéré que ce type d'interaction plante/plante pouvait être également impliqué dans les phénomènes de régénération naturelle des espèces du genre Juniperus. En effet, Fisher \& Gardner (1995) ont observé dans les montagnes d'Oman que la germination de jeunes plants de $J$. excelsia (espèce proche de $J$. thurifera) était assujettie à la présence d'une plante nurse en émettant l'hypothèse que cette dernière matérialisait un environnement favorable à la croissance du jeune plant de Juniperus (sol plus profond, réduction de la température). Chez $J$. thurifera et au Maroc, il a été rapporté que les germinations étaient plus abondantes au sein de touffes de xérophytes épineux en coussinet (Montès 1999). Toutefois le rôle de la symbiose mycorhizienne dans ce type d'interaction Juniperus/plante nurse n'a pas été mentionné.

\section{Conclusion}

Malgré le peu de connaissances scientifiques acquises sur le rôle de la symbiose mycorhizienne dans le développement des espèces de Juniperus et plus particulièrement dans les phénomènes de régénérations naturelles, les quelques résultats disponibles permettent d'encourager l'introduction et la valorisation de la composante mycorhizienne dans les itinéraires culturaux à mettre en place pour conserver durablement les thuriféraies sur le pourtour méditerranéen au nord comme au sud. Afin d'atteindre cet objectif, il sera nécessaire d'entreprendre des études pour :

- décrire la structure des communautés fongiques composant le cortège mycorhizien à différents stades de développement de l'arbre ;

- analyser le rôle écologique et fonctionnel de la double symbiose (symbiose ectomycorhizienne/symbiose mycorhizienne à arbuscules) tant au niveau de la croissance de l'arbre que de son impact sur le biofonctionnement du sol ;

- évaluer le rôle de la flore accompagnatrice du Genévrier thurifère dans les phénomènes de régénération naturelle et plus particulièrement au niveau de son impact sur l'évolution spatio-temporelle des communautés 
de champignons mycorhiziens. L'identification des plantes nurses les plus performantes permettra d'établir des plans de gestion de la strate arbustive présente dans les formations à Genévrier thurifère ;

- entreprendre des opérations de mycorhization contrôlée dans les milieux les plus dégradés afin de contribuer à la valorisation et la conservation de cette essence forestière en la réintroduisant dans les zones où elle $\mathrm{a}$ été éradiquée.

\section{Références}

Adjoud-Sadadou D. \& Halli-Hargas R., 2000. Occurrence of arbuscular mycorrhiza on aged Eucalyptus. Mycorrhiza 9: 287-290.

Albaladejo J., Martinez-Mena M., Roldan A. \& Castillo V., 1998. Soil degradation and desertification induced by vegetation removal in a semiarid environment. Soil Use Manag. 14: 1-5.

Barea J.M., Azcon-Aguilar C. \& Azcon R., 1997. Interactions between mycorrhizal fungi and rhizosphere micro-organisms within the context of sustainable soil-plant systems. In: Gange A.C., Brown V.K. (eds.), Multitrophic Interactions in TerrestrialSystems. Cambridge, Blackwell Science: 65-77.

Bashan Y., Salazar B., PuenteMa E., Bacilio M. \& Linderman R., 2009. Enhanced establishment and growth of giant cardon cactus in an eroded field in the Sonoran Desert using native legume trees as nurse plants aided by plant growth-promoting microorganisms and compost. Biol. Fert. Soils 45: 585-594.

Bethlenfalvay G.J. \& Linderman R.G., 1992. Mycorrhizae in Sustainable Agriculture. Madison, ASA Special Publication, WI, $124 \mathrm{p}$.

Cázares E. \& Trappe J.M., 1993. Vesicular endophytes in roots of the Pinaceae. Mycorrhiza 2: 153-156.

Cázares E. \& Smith J.E., 1996. Occurrence of vesicular-arbuscular mycorrhizae in Pseudotsuga menziesii and Tsuga heterophylla seedlings grown in Oregon Coast Range soils. Mycorrhiza 6: 65-67.

Dhillion S.S., 1994. Ectomycorrhizae, arbuscular mycorrhizae, and Rhizoctonia sp. of alpine and boreal Salix spp. in Norway. Arct. Alp. Res. 26: 304307.

Duponnois R., Plenchette C. \& Bâ A.M., 2001. Growth stimulation of seventeen fallow leguminous plants inoculated with Glomus aggregatum in Senegal. Eur. J. Soil Biol. 37: 181-186.

Duponnois R., Plenchette C., Prin Y., Ducousso M., Kisa M., Bâ A.M. \& Galiana A., 2007. Use of mycorrhizal inoculation to improve reafforestation process with Australian Acacia in Sahelian ecozones. Ecol. Eng. 29: 105-112.

Duponnois R., Ouahmane L., Kane A., Thioulouse J., Hafidi M., Boumezzough A., Prin Y, Baudoin E., Galiana A. \& Dreyfus B., 2011. Nurse shrubs increased the early growth of Cupressus seedlings by enhancing below ground mutualism and microbial soil functionalities. Soil Biol. Biochem. 43: 2160-2168.
Fisher M. \& Gardner A.S., 1995. The status and ecology of a Juniperus excelsia subsp. polycarpus woodland in the northern mountains of Oman. Vegetatio 119: 33-51.

Founoune H., Duponnois R. \& Bâ A.M., 2002. Influence of the dual arbuscular endomycorrhizal / ectomycorrhizal symbiosis on the growth of Acacia holosericea in glasshouse conditions (A. Cunn. ex G. Don). Ann. For. Sci. 59: 93-98.

Francis D.F. \& Thornes J.B., 1990. Matorral: erosion and reclamation. In: Albaladejo J., Stocking M.A., Diaz E. (eds.), Soil Degradation and Rehabilitation in Mediterranean Environmental Conditions. CsIC, Spain, Murcia: 87-115.

Garcia C., Hernandez T., Roldan A. \& Albaladejo L., 1997. Biological and biochemical quality of a semiarid soil after induced revegetation. J. Environ. Qual. 26: 1116-1122.

Garner W. \& Steinberger Y., 1989. A proposed mechanism for the formation of fertile islands in the desert ecosystem. J. Arid Environ. 16: 257-262.

Gauquelin T., Bertaudière V., Montes N., Badri W. \& Asmodé J.F., 1999. Endangered stands of Thuriferous juniper in the western Mediterranean basin: Ecological status, Conservation and Management. Biodivers. Conserv. 8 (11): 1479-1498.

Habte M. \& Manjunath A., 1991. Categories of vesicular-arbuscular mycorrhizal dependency of host species. Mycorrhiza 1: 3-12.

Lapeyrie F.F. \& Chilvers G.A., 1985. An endomycorrhiza-ectomycorrhizasuccession associated with enhanced growth of Eucalyptus dumosa seedlings planted in a calcareous soil. New Phytol. 100: 93104.

Lodge D.J., 1989. The influence of soil moisture and flooding information of VA-endo- and ectomycorrhizae in Populus and Salix. Plant Soil 117: 243-253.

Lodge D.J. \& Wentworth T.R., 1990. Negative associations among VA-mycorrhizal fungi and some ectomycorrhizal fungi inhabiting the same root system. Oikos 57: 347-356.

Molina R., Myrold D. \& Li C.Y., 1994. Root symbiosis of red alder: technological opportunities for enhanced regeneration and soil improvement. In: Hibbs D.E., DeBell D.S., Tarrant R.F. (eds), The biology and management of red alder. Corvallis, Ore., Oregon State University Press: 23-46.

Montes N., 1999. Dynamique et gestion d'une formation arborée à Genévrier thurifère (Juniperus thurifera L.) des Atlas marocains : le cas de la vallée de 1'Azzaden. Thèse Université Toulouse 2, Toulouse, France, $234 \mathrm{p}$.

Odum E.P., 1959. Fundamentals of Ecology. Philadelphia, Saunders, $546 \mathrm{p}$.

Ouahmane L., Hafidi M., Kisa M., Boumezzouch A., Thioulouse J. \& Duponnois R., 2006. Lavandula species as a source of arbuscular mycorrhizal propagules facilitating the early development of Cupressus arizonica. Appl. Soil Ecol. 34: 190-199.

Ouahmane L., Hafidi M., Thioulouse J., Ducousso M., Kisa M., Prin Y., Galiana A., Boumezzough A. \& Duponnois R., 2007. Improvement of Cupressus atlantica Gaussen growth by inoculation with native arbuscular mycorrhizal fungi. J. Appl. Microbiol. 103: 683-690.

Plenchette C., Fortin J.A. \& Furlan V., 1983. Growth responses to several plant species to mycorrhizae in a soil of moderate P-fertility. I. Mycorrhizal dependency under field conditions. Plant Soil 70: 199-209. 
Requena N., Perez-Solis E., Azcon-Aguilar C., Jeffries P. \& Barea J.M., 2001. Management of indigenous plant-microbe symbioses aids restoration of desertified ecosystems. Appl. Environ. Microbiol. 67: 495-498.

Roldan A., Carrasco L. \& Caravaca F., 2006. Stability of dessicated rhizosphere soil aggregates of mycorrhizal Juniperus oxycedrus grown in a desertified soil amended with a composted organic residue. Soil Biol. Biochem. 38: 2722-2730.

Schreiner R.P., Mihara K.L., McDaniel H. \& Bethlenfalvay G.J., 1997. Mycorrhizal fungi influence plant and soil functions and interactions. Plant Soil 188: 199-209.

Smith S. \& Read J., 2008. Mycorrhizal symbiosis. Ed. Hardcover, $800 \mathrm{p}$.

Stahl E., 1900. Der Sinn der Mycorhizenbildung. Jahrb. Wiss. Bot. 34: 539-668.
Valladares F. \& Pugnaire F.I., 1999. Tradeoffs between irradiance capture and avoidance in semiarid environments assessed with a crown architecture model. Ann. Bot. 83: 459-469.

Van der Heijden M.G.A., 2001. Differential benefits of arbuscular mycorrhizal and ectomycorrhizal infection of Salix repens. Mycorrhiza 10: 185-193.

Van der Hejden M.G.A., Klironomos J.N., Ursic M., Moutoglis P., Streitwolf-Engel R., Boller T., Wiemken A. \& Sanders I.R., 1998. Mycorrhizal fungal diversity determines plant biodiversity, ecosystem variability and productivity. Nature 396: 69-72.

Van der Heijden M.G.A. \& Vosatka M., 1999. Mycorrhizal associations of Salix repens L. communities in succession of dune ecosystems. II. Mycorrhizal dynamics and interaction of ectomycorrhizaland arbuscular mycorrhizal fungi. Can. J. Bot. 77: 18331841. 